Relations industrielles

Industrial Relations

\title{
The Basic Barnard, by William B. Wolf, Ithaca, New York State School of Industrial and Labor Relations, Cornell University, 1974, 140 pp.
}

\section{Laurent Bélanger}

Volume 29, numéro 3, 1974

URI : https://id.erudit.org/iderudit/028545ar

DOI : https://doi.org/10.7202/028545ar

Aller au sommaire du numéro

Éditeur(s)

Département des relations industrielles de l'Université Laval

ISSN

0034-379X (imprimé)

1703-8138 (numérique)

Découvrir la revue

Citer ce compte rendu

Bélanger, L. (1974). Compte rendu de [The Basic Barnard, by William B. Wolf, Ithaca, New York State School of Industrial and Labor Relations, Cornell

University, 1974, 140 pp.] Relations industrielles / Industrial Relations, 29(3),

626-626. https://doi.org/10.7202/028545ar

Tous droits réservés (C Département des relations industrielles de l'Université Laval, 1974
Ce document est protégé par la loi sur le droit d'auteur. L'utilisation des services d’Érudit (y compris la reproduction) est assujettie à sa politique d'utilisation que vous pouvez consulter en ligne.

https://apropos.erudit.org/fr/usagers/politique-dutilisation/ 
The Basic Barnard, by William B. Wolf, Ithaca, New York State School of Industrial and Labor Relations, Cornell University, 1974, $140 \mathrm{pp}$.

Dans cet ouvrage d'un peu plus de cent pages, William Bernard Wolf tente de tracer un profil de Chester I. Barnard, un auteur bien connu en administration des entreprises surtout par son ouvrage: The Functions of the Executive, Cambridge University Press, 1938. Après avoir colligé les conférences et les manuscrits de C.I. Barnard et à la suite d'une série d'entrevues avec ce dernier avant son décès, Wolf nous présente les notions de base qui ont servi à C.I. Barnard tant dans l'élaboration de son ouvrage que dans l'exercice des fonctions qu'il a remplies au cours de sa carrière. On se rappelle que Barnard a occupé pendant plus de vingt ans le poste de la présidence à la New Jersey Bell Telephone Co. Il était aussi durant la guerre à la tête d'une organisation importante, la United Service Organization (U.S.O.). Tout en étant un praticien de l'administration, C.I. Barnard a réussi à étaborer une théorie de l'organisation et du management qu'il voulait d'une application la plus générale possible. En définissant une organisation commẹ un système d'activités de deux ou plusieurs personnes consciemment coordonnées en vue de réaliser des objectifs communs, Barnard puisa largement dans la théorie générale de Pareto. Il a été ainsi amené à utiliser l'approche structuro-fonctionnelle dans le description de la structure et du fonctionnement d'une organisation. Il s'est fait d'abord une représentation intuitive de l'organisation qu'il a réussi par la suite à étayer par des leçons tirées de son expérience personnelle. Barnard a été ainsi un des premiers auteurs à traiter dans un même ouvrage des dimensions formelles et informelles de l'organisation, du fondement de l'autorité, de la prise de décision et des communications. C'est surtout le thème de l'autorité dans l'entreprise qui a été et qui est encore la pierre d'achoppement des étudiants en administration. Pour Barnard, l'autorité réside avant tout dans le caractère d'une communication ou d'un ordre. L'autorité, pour reprendre une expression plus connue, vient d'en bas, c'est-à-dire qu'elle repose sur l'acceptation consciente de la part de l'individu qui reçoit cette communication et qui peut difficilement la contester puisqu'il est toujours possible de réaliser qu'une communication est reliée à la poursuite d'objectifs organisationnels. Cette théorie de l'autorité devient plus compréhensible si l'on connaît la conception que Barnard se fait de l'individu dans la société et dans les organisations. L'auteur de cet ouvrage consacre une section à la philosophie sociale de Barnard. Ce passage nous permet de saisir la manière dont Barnard traite du dilemme "individu et organisation 》, un thème repris par la suite par Chris Argyris. Tout en reconnaissant chez l'individu la légitimité de la poursuite d'aspirations personnelles, Barnard soutient qu'un système persiste par la volonté de collaboration des membres. Cette collaboration s'obtient soit par la force, par des incitations d'ordre monétaire ou psychologique, ou encore, par la persuasion. Qu'on utilise l'une ou l'autre des méthodes, les buts communs de toute organisation ont préséance sur les buts particuliers ou les aspirations personnelles des gens. En reliant cette vision de l'homme et de l'organisation à celle de la notion d'autorité, on peut saisir plus facilement l'idée d'acceptation «consciente » chez les personnes qui doivent exécuter des ordres. Dans une telle perspective, les possibilités de contestation individuelle de l'autorité sont plutôt minces. Wolf, dans ce volume, ne veut pas aborder d'une façon critique la théorie de Barnard et se garde bien de remettre en cause les fondements conceptuels qui ont servi à son élaboration. Malgré cette lacune, l'ouvrage de Wolf m'apparaît nécessaire à une bonne compréhension de l'oeuvre de Chester I. Barnard, qui demeure toujours difficile d'accès à ceux qui s'intéressent au fonctionnement des organisations, à cause de son caractère abstrait et de son manque de clarté.

\section{Laurent BELANGER}

Université Laval 